\title{
A Perceptual Study into the Behaviour of Autonomous Agents within a Virtual Urban Environment
}

\author{
Stuart O'Connor, Fotis Liarokapis \\ Interactive Worlds Applied Research Group \\ Coventry University \\ Coventry, UK \\ oconno13@uni.coventry.ac.uk, \\ F.Liarokapis@coventry.ac.uk
}

\author{
Christopher Peters \\ KTH Royal Institute of Technology \\ HPCViz \\ Sweden \\ chpeters@kth.se
}

\begin{abstract}
Simulating vast crowds of autonomous agents within a procedurally generated virtual environment is a challenging endeavour from a technical perspective, however it becomes even more difficult when the subjective nature of perception is also taken into account. Agent behaviour is the product of artificial intelligence systems working in tandem, however the sophistication of these systems is not a guarantee of achieving believable behaviour. Within locations based upon reality such as an urban environment, the perceived realism of agent behaviour becomes even harder to achieve. This paper presents the development of a crowd simulation that is based upon a real-life urban environment, which is then subjected to perceptual experimentation to identify features of behaviour which can be linked to perceived realism. This research is predicted to feedback into the development processes of inhabited cities, especially those attempting to simulate perceptually realistic agents as it will highlight features of behaviour that are important to implement. The perceptual experimentation methodologies presented can also be adapted and potentially utilised to test other types of crowd simulation, whether it be for the purposes of computer games or even urban planning and health and safety.
\end{abstract}

Keywords-crowd simulation, perceptual studies, artificial intelligence, agent behaviour, virtual urban environment

\section{INTRODUCTION}

Crowd simulation is the process of populating a virtual scene with a large number of intelligent agents that display distinct collective behaviours. Crowd simulations have different purposes, such as for serious applications including evacuation scenarios and for entertainment including computer games. Whilst serious applications require a simulation to have virtual realism and be as close to reality as possible, other types especially for entertainment require perceived realism and a high level of plausibility. Recent research has started to consider this plausibility within predefined environments [1] however few studies consider the plausibility in terms of the perceived realism of agent crowd behaviour. This paper gives an overview of the types of perceptual tests that can be utilised to gauge the perceived realism of agent crowd behaviour within a virtual simulation. These methods go towards finding a solution to this research question: 'How to evaluate the perceived realism of agent crowd behaviour within a virtual urban environment'.

A virtual scene is generally based upon a real-life counterpart in some way, shape, or form [2]. A famous example for crowd simulation is the Shibuya Crossing in Tokyo. Agents are expected to behave in a manner not dissimilar from a real person within the same context [3], and even though a simulation may be implemented to be virtually realistic, perceptually that may not be the case. It is highly important that a crowd simulation is perceived to be realistic by human viewers or else plausibility will be lost, which is especially true for computer games that require a level of immersion [4]. This research will investigate the perceived realism of agent behaviour within an urban environment through perceptual experimentation, in order to identify features of behaviour that are the most effective for ensuring the perceptual plausibility of the virtual scene.

This paper is organised as follows. Section II, presents related research and methods on crowd simulation techniques and the evaluation of crowds. The development and implementation of the crowd simulation within a virtual urban environment is detailed in section III. Section IV illustrates the methodologies for the perceived realism experiments, along the results from a preliminary pilot study. Finally, section V provides analysis and conclusions, as well as the direction of future research.

\section{BACKGROUND}

This research relates to two distinct domains: crowd simulation techniques and the evaluation of crowds.

\section{A. Crowd Simulation Techniques}

The real-time simulation of crowds has been conducted using a variety of approaches [5], [6]. The most common method is to employ a series of models and algorithms working in tandem to animate each agent. Generally this includes implementing decision making [7], pathfinding navigation [8] augmented with local steering mechanics [9] and an agent 
perception system [10]. Advanced behaviour altering aspects such as agent learning [11], social forces models [12], physiological factors [13] and sociological effects [14] can also be utilised to enhance agent believability. Technologies from the games industry and middleware have been deployed for simulating crowds within a variety of different scenarios, to allow for unique research into specific areas such as safety [2]. The crowd simulation produced as part of this research follows the general build by utilising decision making, pathfinding, steering and perception along with an additional feature, behavioural annotation.

The decision making system is a highly important aspect in any artificial intelligence system [7], as it allows for a choice to be made in order for a specific behaviour or action to be selected from a range of possible behaviours or actions. Next, it depends on the decision making system to discern which of these is the most appropriate to choose at that given moment. There are a number of different decision making systems that can be implemented [15] such as: decision trees, finite state machines and artificial neural networks. Each type has its own advantages and disadvantages (depending upon the requirements of the system). For the purposes of this research, a finite state machine was selected as the most viable solution due to the limited number of states required to achieve the desired crowd behaviours.

The main purpose of pathfinding is to move an agent from its current location to the next selected location (which is often passed down from the decision making processes). Whilst it might not seem like much of a challenge to begin with it can be a difficult process to implement, often due to the complexity and scale of the simulated environment [8]. Pathfinding systems are typically based on graph searching algorithms in the fact that environment is generally split into a number of connected nodes that the algorithm is then applied to. The path between an agent's current location and its destination is calculated but the manner of how this occurs depends upon the implemented algorithms. Common pathfinding algorithms include: best-first search, Dijkstra's and $A^{*}$ [16]. In this work, $\mathrm{A}^{*}$ was selected because it is capable of finding a path if there is a possible path to find (whereas other algorithms are not guaranteed to always find a path even if there is one). This allows for the possibility of multiple environmental configurations.

Steering mechanics are a common component for agent systems, especially those implemented within simulations and computer games. Steering allows an agent to follow specific behaviours that alter its local movement in some manner, depending upon which steering mechanic has been implemented. The type of steering mechanic applied most often depends upon the given scenario and the type of agents being simulated. Most all steering mechanics utilise some kind of perception system as this is allows them to access an agents local environment for specific conditions. The sophistication of the perception system is generally dictated by the other artificial intelligence and behavioural features available to utilise the local data.

A common approach for agent perception is to designate a radius around the agent to act as its locally accessible neighbourhood. This is the type of perception implemented for the crowd simulation developed as part of this research, as it is effective for both the steering an annotation systems. Common steering mechanics [17] include flocking, seek and flee, obstacle avoidance and path following. In this work, crowd simulation path-following was implemented due to the nature of human crowds and there affinity for following the main paths situated within the environment.

Most crowd behaviour techniques require specific scripting for the agents [18] however behavioural annotation works differently by embedding the information within specific features in the virtual environment [3], [19]. Most behaviour altering methods such as agent learning [11] embed the information within the autonomous agents themselves. This can be costly in terms of complexity and processing but by utilising the behavioural annotation mechanism this can potentially be reduced. Agents perceive these annotations within the virtual environment [15] using their individual perception systems. They then alter their own behaviour as their decision making systems take into account the feature they have just detected. Additional information, such as advanced feature descriptions or local magnitude can be stored to further tailor agent behaviour to their environment. Behavioural annotation is a feature implemented within the crowd simulation developed in this research, due to its versatile nature of being able to add behaviours depending upon the environment.

\section{B. Evaluation of Crowds}

The evaluation of crowds can be completed using a variety of methods to check different factors ranging from agent appearance and animation to behaviour [20]. Research has been conducted into evaluating crowd simulations by comparing the virtual scenes to reality [21], [22]. By looking for specific local spatial or temporal crowd patterns [23], comparing crowd densities and using visual comparison, a good indication of whether a simulation is close to reality can be obtained. There has also been research regarding evaluating the motion of individuals [24], groups [25] and crowds [1] of agents based on human perception. Often these studies search for a level of detail that provides a balance between perceptual realism and performance [26], sometimes by comparing specific features that are present within the simulation, such as collision detection [27]. These perceptual evaluation methodologies are similar to the types of psychophysical perceptual experiments suggested as part of this research.

Furthermore, the constant stimuli procedure is a classical psychophysical methodology where participants are asked to perceptually evaluate a stream of different magnitudes of stimulus that are not presented in a given order such as ascending or descending, but instead randomly shown. This is a useful approach because it allows to limit participants from actively predicting the next level of stimulus (as is common with some others methods and in turn reduces errors from habituation and expectation). Full sampling of the psychometric function can be achieved with this methodology and a threshold value can be gained, however if multiple parameters are altered for perceptual purposes it can result in a lot of trails. 
Moreover, the adjustment procedure is a type of classical psychophysical methodology where participants take control of the magnitude of the stimulus to identify the detectable threshold for the specific feature. This process is repeated a number of times to ensure the threshold for detection of the feature is the same against varying other background features. In this manner, a mean value can be calculated for the average error that in turn can be utilised to identify the threshold sensitivity.

In addition, a staircase procedure is an adaptive type of psychophysical methodology in which the stimulus is constantly adapted to the individual participant. These types of psychophysical procedures are suggested to yield more accurate results than classical methodologies [28]. The general procedure for a staircase method is to start at a high magnitude stimulus and then reduce the stimulus until the participant can notice the change, at which point there is a reversal of the staircase and the stimulus is increased until the participant notices again. This can be repeated so that the threshold values for these reversals can be averaged to gain more reliable results. Staircase procedures can vary depending upon type and what is being perceptually measured. Due to the increased reliability of the adaptive type of procedure, the staircase methodology is the main psychophysical perceptual experiment to be utilised in this research to gauge the threshold values for the simulations features.

\section{CROWD SIMULATION}

The main purpose for developing the urban crowd simulation was to create a platform with alterable parameters and variables capable of transforming agent behaviour for the purposes of experimental evaluation. Due to the primary aim being probing human impressions, the standard modelling and behaviour approaches had to be altered to accommodate the fact that more stimuli were needed than just the configuration that appeared most realistic to the developer. These studies are expected to feed back into informing the development process, allowing the parameters and variables of the simulations features to be automatically set to maximise viewer impressions of plausibility.

There are three distinct elements in the development process of the urban crowd simulation including the urban environment for the generation of the virtual location; the agent simulation for the real-time simulation of crowds of agents; and the behavioural annotation for annotating features within the virtual environment to provide a basis for supporting crowd AI behaviour.

\section{A. Urban Environment}

The first phase of development for the simulation was to create the urban environment so that it could later be populated with smart agents. A highly populated urban location was selected and a birds-eye view image of the area was imported from Google maps. This image was then bound to a plane to act as a two-dimensional backdrop for the virtual scene. Using this backdrop pathway, it can be identified, along with buildings and other features for impacting the simulation of agents. The emphasis is primarily on creating a virtual environment that is viable for simulating crowd behaviour within an urban setting rather than graphical fidelity (i.e. complex meshes, lighting and shading).

\section{B. Agent Simulation}

All implementation efforts are being conducted using $\mathrm{C}++$ and the cross-platform OpenGL graphics library for visualisation. At run-time, simulating large crowds of agents requires constant updating frame by frame as shown in Fig. 1.

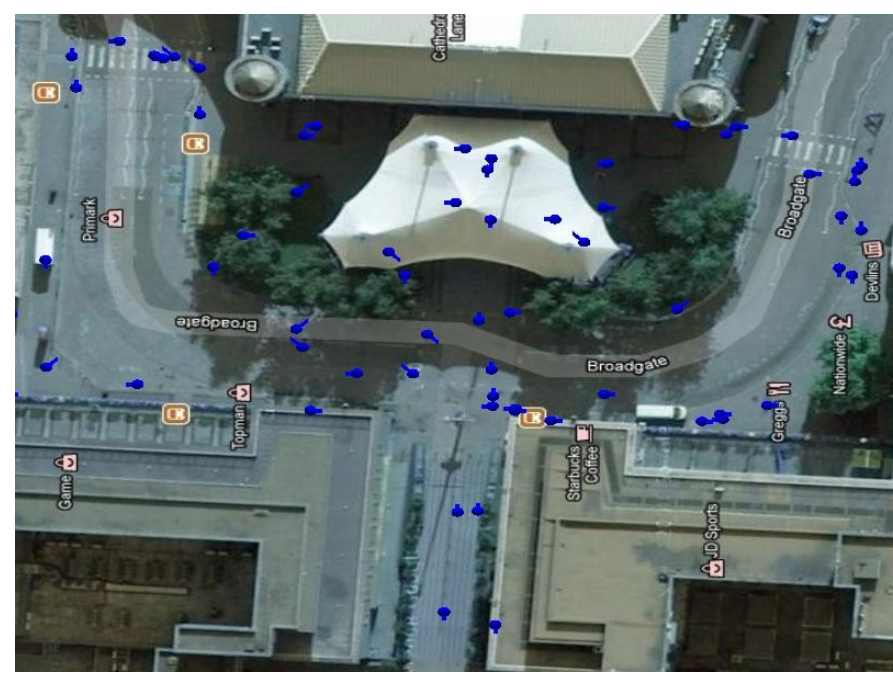

Fig. 1. An exemplar of the urban crowd simulation at run-time with the agents visible. The backdrop can be seen which is a bird's eye view of Coventry city centre in England

Each agent has its own data structure including a number of variables for purposes varying from movement to behaviour orientation. This agent data structure allows for the adaptation and altering of specific agent features which can then be utilised for evaluation purposes. The main implemented artificial intelligence techniques include, finite state machines, $\mathrm{A}^{*}$ pathfinding, path-following steering and radial perception. When the environment is generated a structure of waypoints and cells are also initialised to provide environmental and navigational data for agents. When the simulation is ran, each agent is also initialised and set with key data regarding its coordinates, cell position, vector and other essentials. The major initialisation steps for setting up an agent are as follows.

At initialisation an agent is given an ID of $\mathrm{N}$ (with $\mathrm{N}$ being the number of the current agent iteration). Next the agents' variables are set given, $\mathrm{N}$ multiplied by agent factor, with agent factor being a random element populated for each agent. These values are then processed for each agent variable to give usable values. Finally the agents' waypoint number is calculated by the agents position divided by the waypoint locations, to discover which waypoint the agent is in close proximity too. This becomes the waypoint number which is utilised for the movement routine. Each frame is calculated as follows:

- The agent checks its local area via its perceptual radius for annotation to alter its behaviour. 
- Next the movement routine begins. If the agents path finished variable is equal to false then the next movement along its currently held path is calculated.

- The agents' normalised vector is calculated from the agents' current position and the position of its next waypoint.

- The agents steering is taken into account, calculated as agent normalised vector multiplied by the agent steering value.

- Then the agent is moved along its path by one step, calculated as agent position equals agent velocity multiplied by agent normalised vector.

- The agents' position is then compared to the position of its destination and if this has been reached the path finished variable is altered to true.

- When the movement routine begins if the path finished variable is false then the decision making system is called upon to select a new destination given the agents individual variable values and the current environmental factors within close proximity. This destination will vary from agent to agent.

- The destination is then passed to the pathfinding $\mathrm{A}^{*}$ algorithm which finds a path in the form of a list of waypoints from the agents current location to its destination. This change the path finished variable to 'false'.

\section{Behavioural Annotation}

In the simulation an underlying grid of cells is initialised in addition to the waypoints utilised for navigation. It is in these cells that the behavioural annotation data is housed (see Fig. 2.), mainly as Boolean values for denoting if an environmental feature is present however more data can be added if necessary such as a numeric value for the intensity of a certain feature.

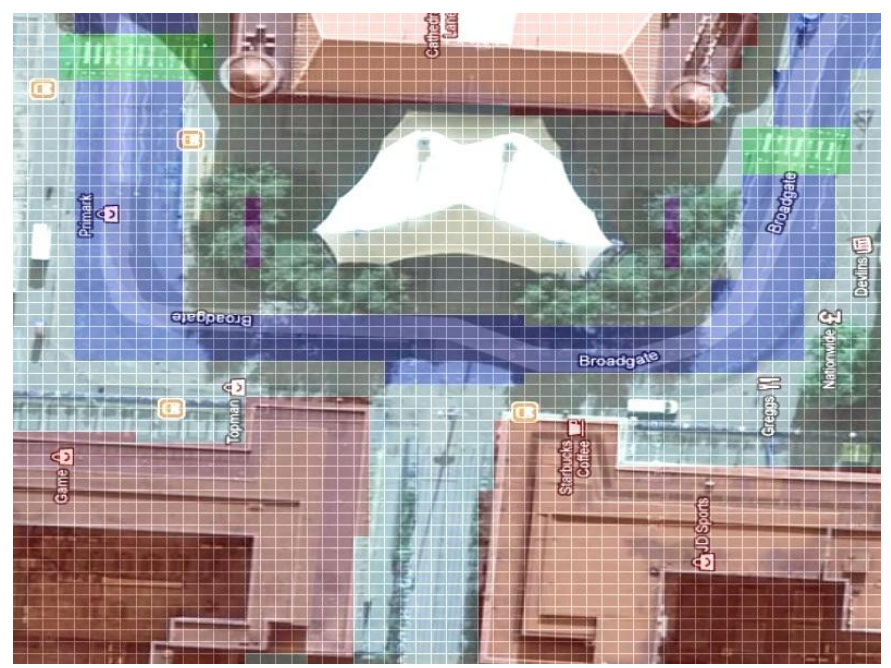

Fig. 2. A screenshot of the urban crowd simulation showing the annotation mechanism visually represented within the underlying grid. Green cells show pedestrian crossing, blue cells show roads and purple cells show stationary positions
The agents detect these annotated cells using the individual perception systems, which is a perceptual radius. In an agents' update routine the annotation is searched for as shown below:

- The agents checks it local area within its perceptual radius.

- If an annotated cells is discovered its contents are read.

- If an annotated feature is found to be true within the cell, depending upon the type of feature present the agent will alter its internal variables. This can have a number of effects including altering the agents' velocity, behaviour type and even its decision making processes so that it selects a different destination.

- Whether an annotation was found or not the agent will then continue with the rest of its update routine.

The annotated features present within the simulation can be turned on or off to give configurations of full annotation, partial annotation or no annotation. Full annotation includes all annotated features, partial annotation contains a few annotated features and no annotation has the mechanism turned completely off. It is hoped that the addition of these annotations will provide a clearer relationship between the agents and their local environment, which can be tested during evaluation.

\section{PERCEPTUAL EXPERIMENT}

At the core of the method are the perceptual experiments that permit the exploration of the parameter and variable spaces implemented into the crowd simulation. As the main purpose is to evaluate the plausibility of the simulation's visual content, the aim is to identify the combinations of parameters and variables that produce the most credible virtual scene. The results of these experiments will be utilised to fine tune the agents and algorithms, as well as highlight new areas or features that could be implemented to maximise the simulation's plausibility. In this manner of synthesis the urban crowd simulation will evolve and become more complex as the research continues.

\section{A. General Methodology}

There are three distinct aspects to the general methodology, analysis, synthesis and perception [1]. These form a cycle that allows for the development and refinement of a crowd simulation with distinct features that can be perceptually evaluated:

- Analysis: Identify features and inform algorithm construction by analysing real-world and similar instances of crowd behaviour.

- Synthesis: Utilise the results of analysis and previous perceptual experiments to synthesise a new generation simulation with more sophisticated features and refinement.

- Perception: Conduct the psychophysical experiments for gauging the perceived realism of the updated simulations features against the older configurations, allowing for the identification of perceptual thresholds. 
The output stimulus produced is a number of short video clips, each showing different configurations of features and parameters all within the same virtual scene. Development of a customised online survey platform for quantitative evaluation utilising large groups of participants is under-way. A prototype that can be utilised for small studies has already been deployed for a pilot study, with the aim of fine tuning the experiments and the psychophysical model. The results of the evaluations conducted as part of the overall methodology feed-back into the simulations features and algorithms until a threshold is reached, whereby a niche in the parameter space is identified that produces visuals that are perceived to be the most credible.

\section{B. Perceived Realism Experiments}

As described in [29] "The art of psychophysics is to formulate a question that is precise and simple enough to obtain a convincing answer', such that it is possible to study the perceptual effects of particular physical dimensions. To this end it is possible to investigate the limits of visual perception by parametrically varying the features within a virtual scene, with the aim to measure the threshold below which realistic plausibility is lost. To measure the perceived realism of the simulation and it's various features a series of experiments have been devised utilising perceptual evaluations.

The data from these experiments will be examined to discover which features and parameters of the simulation are perceived to improve scene realism, so that they can be ranked and an optimal configuration can be discovered. Each participant is shown a series of video clips that contain different versions of the same scene and are asked if it is perceptually realistic. The order and individual configuration of the shown video clips depends upon the experiment being preformed. Three key experiments have been planned:

- The first experiment utilises the staircase procedure to establish the perceptual threshold values of the parameters spaces of each feature within the simulation. This is an important consideration as it will allow for the discovery of the optimal configuration for the simulations features; as such the accuracy of an adaptive psychophysical method has been selected.

- The second experiment utilises the constant stimuli procedure to establish if there is a specific threshold for the number of features required before the simulation is perceived to be realistic.

- The third experiment utilises the adjustment procedure to rank the features based on the perceptual effectiveness. This is an extension for the second experiment to establish if certain features are more effective at achieving perceptual plausibility within a virtual scene.

Initial experiments consider variations in agent movement velocity as the primary feature. There are two main conditions for velocity, uniform velocity and varying velocity. In uniform velocity all agents move at the same rate whereas in varying velocity agents move at different rates. These two conditions have parameter spaces such as range for the varying velocity agents and these can be perceptually evaluated. As the research continues more features will be identified and added to enhance the simulation as is outlined within the overall methodology, so that more psychophysical experimentation can take place.

\section{Perceived Realism Pilot Study}

Using the first experimental method that utilises the staircase procedure as a basis, a pilot study was conducted with three participants in order to test the experiments viability for larger statistically valid samples. The general consensus of the results showed that the participants perceived the simulation to be more realistic if the configuration included the varying velocity feature. Whilst this is not a fair representation due to the small test pool it does follow the general prediction for what one would expect as in reality people do move at different velocities. Parameter space values were also determined for the range, maximum and minimum velocities. Velocity range normalised was 0.3 . Velocity range normalised thresholds were 0.2 and 0.5 . Again whilst not statistically valid this shows that the experiment can produce viable results capable of finding the thresholds and parameter space values of the simulations features. This pilot study has provided preliminary information on the viability of the experimental methodology and suggests that using a larger participant test pool could yield useful results for computational modelling purposes based on perceived realism.

\section{CONCLUSIONS AND FUTURE WORK}

In this paper, details have been presented regarding research towards investigating the behaviour of autonomous agents within a virtual urban environment through the perceptual evaluation of simulation and agent features. Whilst the modelling methods and evaluation results are in the early stages of research, the methodology has been developed and will continue to enhance the efforts made in terms of the simulation and its perceptual evaluation. For each generation of perception the crowd simulation will be synthesised to develop further features and improvements that will give rise to new and more precise data that will help to improve the modelling processes. Currently an automated behavioural annotation mechanism implemented within procedural city generation techniques is being considered, which would allow for the generation of new configurations as opposed to the current method of manual placement. This would allow for more complex and alternative environment generation, leading to a more sophisticated simulation for perceptual experimentation. Perceived realism is important for entertainment and educational purposes where the human participant needs to become immersed so that the goal of simulation can be achieved. By exploring this type of perceptual realism it is hoped that this research could help to streamline the development processes involved in crowd simulation, by indicating features and parameter space values that can be implemented in order to ensure that perceptual plausibility is achieved for agent behaviour.

\section{ACKNOWLEDGMENTS}

The authors would like to thank the Interactive Worlds Applied Research Group (iWARG) members for their support 
and inspiration and Dr. Etienne de Roesch for his invaluable insights into psychophysics.

\section{REFERENCES}

[1] C. Ennis, C. Peters, and C. O' Sullivan, "Perceptual effects of scene context and viewpoint for virtual pedestrian crowds", ACM Transactions Applied Perception, 8(2), Article No. 10, 2011.

[2] O. Szymanezyk, P. Dickinson, and T. Duckett, "Towards Agent-based Crowd Simulation in Airports using Games Technology". Agent and MultiAgent Systems Technologies and Applications, vol. 6682, 2011, pp. 524-533.

[3] C. Peters, C. Ennis, R. McDonnell, and C. O' Sullivan, "Crowds in context: Evaluating the perceptual plausibility of pedestrian orientations", Eurographics Short Papers, 2008, pp. 227-230.

[4] Ubisoft, Assassin's Creed 3, Ubisoft Montreal, (Available at: http://assassinscreed.ubi.com/ac3/en-gb/index.aspx), Accessed at: 02/03/2013.

[5] N. Doulamis, "Evacuation Planning through Cognitive Crowd Tracking", IEEE Int'l Workshop on Workshop on Systems, Signal and Image Processing (IWSSIP), Halkida, Greece, 2009, pp. 1-4.

[6] R. Narain, A. Golas, S. Curtis, and M.C. Lin, "Aggregate dynamics for dense crowd simulation", ACM Transactions on Graphics (TOG) - Proc. of ACM SIGGRAPH Asia 2009, ACM Press, 28(5), Article No. 122, 2009.

[7] L. Luo, S. Zhou, W. Cai, M. Low, and M. Lees, "Toward a Generic Framework for Modeling Human Behaviors in Crowd Simulation", IEEE/WIC/ACM Int'l Joint Conference on Web Intelligence and Intelligent Agent Technology - Volume 02, vol. 2, Washington, DC, USA, 2009, pp. 275-278.

[8] S. Paris, J. Pettre, and S. Donikian, "Pedestrian Reactive Navigation for Crowd Simulation: a Predictive Approach", Computer Graphics Forum Proc. of Eurographics 2007, 26(3), 2007, pp. 665-674.

[9] C.W. Reynolds, "Flocks, herds, and schools: A distributed behavioural model", Proc. of the $14^{\text {th }}$ Annual Conference on Computer Graphics and Interactive Techniques (SIGGRAPH '87), ACM Press, 21(4), 1987, pp. 25-34.

[10] J. Ondej, J. Pettre, A.H. Olivier, and S. Donikian, "A Synthetic-VisionBased Steering Approach for Crowd Simulation”, ACM SIGGRAPH 2010 Papers, ACM Press, Article No. 123, 2010.

[11] W. Shao, and D. Terzopoulos, "Autonomous pedestrians", Proc. of the 2005 ACM SIGGRAPH/Eurographics symposium on Computer Animation (SCA '05), ACM Press, New York, NY, USA, 2005, pp. 1928.

[12] D. Helbing, and P. Molnar, "Social force model for pedestrian dynamics", Physics Review E, 51(5), American Physical Society, 1995, pp. 4282-4286.

[13] N. Pelechano, J.M. Allbeck, N.I. Badler, "Controlling individual agents in high-density crowd simulation", Proc. of the 2007 ACM SIGGRAPH/Eurographics symposium on Computer animation (SCA '07). Eurographics Association, Aire-la-Ville, Switzerland, 2007, pp. 99108.

[14] F. Durupinar, J. Allbeck, N. Pelechano, and N. Badler, "Creating crowd variation with the ocean personality model", Proc. of the $7^{\text {th }}$ Int'l joint conference on Autonomous agents and multiagent systems (AAMAS '08), International Foundation for Autonomous Agents and Multiagent Systems, 2008, pp. 1217-1220.

[15] E. Anderson, "Playing smart - artificial intelligence in computer games", Proc. of zfxCON03 Conference on Game Development, 2003.

[16] X. Cui, and H. Shi, "A*-based Pathfinding in Modern Computer Games", International Journal of Computer Science and Network Security, 11(1), 2011, pp. 125-130.

[17] C. Reynolds, "Steering behaviours for autonomous characters". Proc. of game developers conference, Miller Freeman Game Group, San Francisco, California, 1999, pp. 763-782.

[18] E.F. Anderson, "Scripted smarts in an intelligent virtual environment: behaviour definition using a simple entity annotation language", Proc. of the 2008 Conference on Future Play: Research, Play, Share (Future Play '08). ACM, New York, NY, USA, 2008, pp.185-188.

[19] C. Peters, S. Dobbyn, B. MacNamee, and C. O' Sullivan, "Smart objects for attentive agents", Proc. of the Int'l Conference in Central Europe on Computer Graphics, Visualization and Computer Vision. 2003.

[20] S. Singh, M. Kapadia, G. Reinman, and P. Faloutsos, P. "SteerBench: A benchmark suite for evaluating steering behaviours", Computer Animation and Virtual Worlds - International Workshop Motion in Games (MIG08), John Wiley and Sons Ltd, 20(5), 2009, pp. 533-548.

[21] B. Banerjee, and L. Kraemer, "Evaluation and comparison of multiagent based crowd simulation systems". Agents for games and simulations II, Frank Dignum (eds.), Springer-Verlag, 2011, pp. 53-66.

[22] S.J. Guy, J. van den Berg, et al., "A statistical similarity measure for aggregate crowd dynamics", ACM Transactions on Graphics (TOG), ACM Press, 31(6), Article No. 190, 2012.

[23] S.R. Musse, V.J. Cassol, and C.R. Jung, "Towards a quantitative approach for comparing crowds", Computer Animation and Virtual Worlds, John Wiley and Sons Ltd, 23(1), 2012, pp. 49-57.

[24] R. McDonnell, F. Newell, and C. O' Sullivan, "Smooth movers: Perceptually guided human motion simulation", Proc. of the 2007 ACM SIGGRAPH/ Eurographics symposium on computer animation (SCA 07), 2007, pp. 259-269.

[25] C. Ennis, R. McDonnell, and C. O' Sullivan, "Seeing is believing: Body motion dominates in multi-sensory conversations", ACM Transactions on Graphics (TOG), ACM Press, 29(4), Article No. 19, 2010.

[26] L. Navarro, F. Flacher, and V. Corruble, "Dynamic Level of Detail for Large Scale Agent-Based Urban Simulations", Proc. of the $10^{\text {th }}$ Int'l Conference on Autonomous Agents and Multiagent Systems, International Foundation for Autonomous Agents and Multiagent Systems, 2011, pp. 701-708.

[27] S. Paris, A. Gerdelan, and C. O' Sullivan, "CA-LOD: Collision Avoidance Level of Detail for Scalable, Controllable Crowds", Lecture Notes in Computer Science, Volume 5884/2009, 2009, pp. 13-28.

[28] H. Dai, "On measuring psychometric functions: A comparison of the constant-stimulus and adaptive up-down methods", Journal of the Acoustical Society of America, 98(6), 1995, pp. 3135-3139.

[29] W.H. Ehrenstein, and A. Ehrenstein, "Psychophysical Methods", Modern techniques in neuroscience research, 1999, pp. 1211-1241. 